\title{
Hermite-Hadamard inequality for functions whose derivatives absolute values are preinvex
}

\author{
Ali Barani ${ }^{\text {** }}$, Amir G Ghazanfari ${ }^{1}$ and Sever S Dragomir ${ }^{2,3}$
}

\section{"Correspondence:}

alibarani2000@yahoo.com; barani.a@lu.ac.ir

'Department of Mathematics, Lorestan University, P.O. Box 465, Khoramabad, Iran

Full list of author information is available at the end of the article

\begin{abstract}
In this article, we extend some estimates of the right-hand side of a Hermite-Hadamard-type inequality for preinvex functions. Then, a generalization to functions of several variables on invex subsets of $\mathbb{R}^{n}$ is introduced.
\end{abstract}

Keywords: Hermite-Hadamard inequality; invex sets; preinvex functions

\section{Introduction and preliminary}

Let $I=[c, d]$ be an interval on the real line $\mathbb{R}$, let $f: I \rightarrow \mathbb{R}$ be a convex function and let $a, b \in[c, d], a<b$. We consider the well-known Hadamard's inequality

$$
f\left(\frac{a+b}{2}\right) \leq \frac{1}{b-a} \int_{a}^{b} f(x) d x \leq \frac{f(a)+f(b)}{2} .
$$

Both inequalities hold in the reversed direction if $f$ is concave. We note that Hadamard's inequality may be regarded as a refinement of the concept of convexity and it follows easily from Jensen's inequality. Hadamard's inequality for convex functions has received renewed attention in recent years and a remarkable variety of refinements and generalizations have been found (see, for example, [1-4]).

The classical Hermite-Hadamard inequality provides estimates of the mean value of a continuous convex function $f:[a, b] \rightarrow \mathbb{R}$.

Dragomir and Agarwal [5] used the formula,

$$
\frac{f(a)+f(b)}{2}-\frac{1}{b-a} \int_{a}^{b} f(x) d x=\frac{b-a}{2} \int_{0}^{1}(1-2 t) f^{\prime}(t a+(1-t) b) d t
$$

to prove the following results.

Theorem 1.1 Assume $a, b \in \mathbb{R}$ with $a<b$ and $f:[a, b] \rightarrow \mathbb{R}$ is a differentiable function on $(a, b)$. If $\left|f^{\prime}\right|$ is convex on $[a, b]$ then the following inequality holds true

$$
\left|\frac{f(a)+f(b)}{2}-\frac{1}{b-a} \int_{a}^{b} f(x) d x\right| \leq \frac{(b-a)\left(\left|f^{\prime}(a)\right|+\left|f^{\prime}(b)\right|\right)}{8} .
$$

\section{Springer}

(c) 2012 Barani et al.; licensee Springer. This is an Open Access article distributed under the terms of the Creative Commons Attribution License (http://creativecommons.org/licenses/by/2.0), which permits unrestricted use, distribution, and reproduction in any medium, provided the original work is properly cited. 
Theorem 1.2 Assume $a, b \in \mathbb{R}$ with $a<b$ and $f:[a, b] \rightarrow \mathbb{R}$ is a differentiable function on $(a, b)$. Assume $p \in \mathbb{R}$ with $p>1$. If $\left|f^{\prime}\right|^{\frac{p}{p-1}}$ is convex on $[a, b]$ then the following inequality holds true

$$
\left|\frac{f(a)+f(b)}{2}-\frac{1}{b-a} \int_{a}^{b} f(x) d x\right| \leq \frac{b-a}{2(p+1)^{1 / p}} \cdot\left[\frac{\left|f^{\prime}(a)\right|^{\frac{p}{p-1}}+\left|f^{\prime}(b)\right|^{\frac{p}{p-1}}}{2}\right]^{\frac{p-1}{p}} .
$$

Ion [6] presented some estimates of the right-hand side of a Hermite-Hadamard-type inequality in which some quasi-convex functions are involved.

In recent years, several extensions and generalizations have been considered for classical convexity. A significant generalization of convex functions is that of invex functions introduced by Hanson [7]. Weir and Mond [8] introduced the concept of preinvex functions and applied it to the establishment of the sufficient optimality conditions and duality in nonlinear programming. Aslam Noor $[9,10]$ introduced the Hermite-Hadamard inequality for preinvex and log-preinvex functions.

In this article, we generalize the results in [6] for functions whose first derivatives absolute values are preinvex. Also some results for functions whose second derivatives absolute values are preinvex will be given. Now, we recall some notions in invexity analysis which will be used throughout the article (see $[11,12]$ and references therein).

Definition 1.1 A set $S \subseteq \mathbb{R}^{n}$ is said to be invex with respect to the map $\eta: S \times S \rightarrow \mathbb{R}^{n}$, if for every $x, y \in S$ and $t \in[0,1]$,

$$
y+t \eta(x, y) \in S .
$$

It is obvious that every convex set is invex with respect to the map $\eta(x, y)=x-y$, but there exist invex sets which are not convex (see [11]). Let $S \subseteq \mathbb{R}^{n}$ be an invex set with respect to $\eta: S \times S \rightarrow \mathbb{R}^{n}$. For every $x, y \in S$ the $\eta$-path $P_{x v}$ joining the points $x$ and $v:=x+\eta(y, x)$ is defined as follows

$$
P_{x v}:=\{z: z=x+\operatorname{t\eta }(y, x): t \in[0,1]\} .
$$

Definition 1.2 Let $S \subseteq \mathbb{R}^{n}$ be an invex set with respect to $\eta: S \times S \rightarrow \mathbb{R}^{n}$. Then, the function $f: S \rightarrow \mathbb{R}$ is said to be preinvex with respect to $\eta$, if for every $x, y \in S$ and $t \in[0,1]$,

$$
f(y+t \eta(x, y)) \leq t f(x)+(1-t) f(y) .
$$

Every convex function is a preinvex with respect to the map $\eta(x, y)=x-y$ but the converse does not holds. For properties and applications of preinvex functions, see [12, 13] and references therein.

The organization of the article is as follows: In Section 2, some generalizations of Hermite-Hadamard-type inequality for first-order differentiable functions are given. Section 3 is devoted to a generalization to several variable preinvex functions. HermiteHadamard-type inequality for second-order differentiable functions are studied in Section 4 . 


\section{First-order differentiable functions}

In this section, we introduce some generalizations of Hermite-Hadamard-type inequality for functions whose first derivatives absolute values are preinvex. We begin with the following lemma which is a generalization of Lemma 2.1 in [5] to invex setting.

Lemma 2.1 Let $A \subseteq \mathbb{R}$ be an open invex subset with respect to $\theta: A \times A \rightarrow \mathbb{R}$ and $a, b \in A$ with $\theta(a, b) \neq 0$. Suppose that $f: A \rightarrow \mathbb{R}$ is a differentiable function. If $f^{\prime}$ is integrable on the $\theta$-path $P_{b c}, c=b+\theta(a, b)$ then, the following equality holds

$$
\begin{aligned}
& -\frac{f(b)+f(b+\theta(a, b))}{2}+\frac{1}{\theta(a, b)} \int_{b}^{b+\theta(a, b)} f(x) d x \\
& =\frac{\theta(a, b)}{2} \int_{0}^{1}(1-2 t) f^{\prime}(b+t \theta(a, b)) d t .
\end{aligned}
$$

Proof Suppose that $a, b \in A$. Since $A$ is an invex set with respect to $\theta$, for every $t \in[0,1]$ we have $b+t \theta(a, b) \in A$. Integrating by parts implies that

$$
\begin{aligned}
& \int_{0}^{1}(1-2 t) f^{\prime}(b+t \theta(a, b)) d t \\
& \quad=\left[\frac{(1-2 t) f(b+t \theta(a, b))}{\theta(a, b)}\right]_{0}^{1}+\frac{2}{\theta(a, b)} \int_{0}^{1} f(b+t \theta(a, b)) d t \\
& \quad=-\frac{f(b)+f(b+\theta(a, b))}{\theta(a, b)}+\frac{2}{(\theta(a, b))^{2}} \int_{b}^{b+\theta(a, b)} f(x) d x
\end{aligned}
$$

which completes the proof.

Theorem 2.1 Let $A \subseteq \mathbb{R}$ be an open invex subset with respect to $\theta: A \times A \rightarrow \mathbb{R}$. Suppose that $f: A \rightarrow \mathbb{R}$ is a differentiable function. If $\left|f^{\prime}\right|$ is preinvex on $A$ then, for every $a, b \in A$ with $\theta(a, b) \neq 0$ the following inequality holds

$$
\begin{aligned}
& \left|\frac{f(b)+f(b+\theta(a, b))}{2}-\frac{1}{\theta(a, b)} \int_{b}^{b+\theta(a, b)} f(x) d x\right| \\
& \quad \leq \frac{|\theta(a, b)|}{8}\left[\left|f^{\prime}(a)\right|+\left|f^{\prime}(b)\right|\right] .
\end{aligned}
$$

Proof Suppose that $a, b \in A$. Since $A$ is an invex set with respect to $\theta$, for every $t \in[0,1]$ we have $b+t \theta(a, b) \in A$. By preinvexity of $\left|f^{\prime}\right|$ and Lemma 2.1 we get

$$
\begin{aligned}
& \left|\frac{f(b)+f(b+\theta(a, b))}{2}-\frac{1}{\theta(a, b)} \int_{b}^{b+\theta(a, b)} f(x) d x\right| \\
& \quad=\left|\frac{\theta(a, b)}{2} \int_{0}^{1}(1-2 t) f^{\prime}(b+t \theta(a, b)) d t\right| \\
& \quad \leq \frac{|\theta(a, b)|}{2}\left\{\int_{0}^{1}|1-2 t|\left(t\left|f^{\prime}(a)\right|+(1-t)\left|f^{\prime}(b)\right|\right) d t\right\} \\
& \quad=\frac{|\theta(a, b)|}{8}\left\{\left|f^{\prime}(a)\right|+\left|f^{\prime}(b)\right|\right\},
\end{aligned}
$$


where,

$$
\int_{0}^{1}|1-2 t|(1-t) d t=\int_{0}^{1}|1-2 t| t d t=\frac{1}{4} .
$$

Now, we give an example of an invex set with respect to an $\theta$ which is satisfies the conditions of Theorem 2.1.

Example 2.1 Suppose that $K:=(-3,-1) \cup(1,4)$ and the function $\theta: K \times K \rightarrow \mathbb{R}$ is defined by

$$
\theta(x, y)= \begin{cases}x-y, & x>0, y>0, \\ x-y, & x<0, y<0, \\ 3-y, & x<0, y>0, \\ -2-y, & x>0, y<0\end{cases}
$$

Clearly $K$ is an open invex set with respect to $\theta$. Suppose that $a \in(-3,-1)$ and $b \in(1,4)$, $b \neq 3$ hence, $\theta(a, b)=3-b \neq 0$. Now,

$$
P_{b c}=[b, 3], \quad b<3,
$$

and

$$
P_{b c}=[3, b], \quad b>3,
$$

where $c=b+\theta(a, b)$.

Another similar result is embodied in the following theorem.

Theorem 2.2 Let $A \subseteq \mathbb{R}$ be an open invex subset with respect to $\theta: A \times A \rightarrow \mathbb{R}$. Suppose that $f: A \rightarrow \mathbb{R}$ is a differentiable function. Assume that $p \in \mathbb{R}$ with $p>1$. If $\left|f^{\prime}\right|^{p / p-1}$ is preinvex on $A$ then, for every $a, b \in A$ with $\theta(a, b) \neq 0$ the following inequality holds

$$
\begin{aligned}
& \left|\frac{f(b)+f(b+\theta(a, b))}{2}-\frac{1}{\theta(a, b)} \int_{b}^{b+\theta(a, b)} f(x) d x\right| \\
& \leq \frac{|\theta(a, b)|}{2(p+1)^{1 / p}}\left[\left|f^{\prime}(a)\right|^{p / p-1}+\left|f^{\prime}(b)\right|^{p / p-1}\right]^{\frac{p-1}{p}} .
\end{aligned}
$$

Proof Suppose that $a, b \in A$. By assumption, Hölder's inequality and the proof of Theorem 4.1 we have

$$
\begin{aligned}
& \left|\frac{f(b)+f(b+\theta(a, b))}{2}-\frac{1}{\theta(a, b)} \int_{b}^{b+\theta(a, b)} f(x) d x\right| \\
& \quad \leq \frac{|\theta(a, b)|}{2} \int_{0}^{1}|1-2 t|\left|f^{\prime}(b+t \theta(a, b))\right| d t \\
& \quad \leq \frac{|\theta(a, b)|}{2}\left(\int_{0}^{1}|1-2 t|^{p} d t\right)^{1 / p}\left(\int_{0}^{1}\left|f^{\prime}(b+t \theta(a, b))\right|^{q} d t\right)^{1 / q}
\end{aligned}
$$




$$
\begin{aligned}
& =\frac{|\theta(a, b)|}{2(p+1)^{1 / p}}\left(\int_{0}^{1}\left|f^{\prime}(b+t \theta(a, b))\right|^{q} d t\right)^{1 / q} \\
& \leq \frac{|\theta(a, b)|}{2(p+1)^{1 / p}}\left(\int_{0}^{1}\left[t\left|f^{\prime}(a)\right|^{q}+(1-t)\left|f^{\prime}(a)\right|^{q}\right] d t\right)^{1 / q} \\
& =\frac{|\theta(a, b)|}{2(p+1)^{1 / p}}\left[\left|f^{\prime}(a)\right|^{q}+\left|f^{\prime}(a)\right|^{q}\right]^{1 / q},
\end{aligned}
$$

where $q:=p /(p-1)$.

Note that if $A=[a, b]$ and $\theta(x, y)=x-y$ for every $x, y \in A$ then, we can deduce Theorems 1.1 and 1.2, from Theorems 2.1 and 2.2, respectively.

\section{An extension to several variables functions}

The aim of this section is to extend the Proposition 1 in [6] and Theorem 2.2 to functions of several variables defined on invex subsets of $\mathbb{R}^{n}$.

The mapping $\eta: S \times S \rightarrow \mathbb{R}^{n}$ is said to be satisfies the condition $C$ if for every $x, y \in S$ and $t \in[0,1]$,

$$
\begin{aligned}
& \eta(y, y+t \eta(x, y))=-t \eta(x, y), \\
& \eta(x, y+t \eta(x, y))=(1-t) \eta(x, y) .
\end{aligned}
$$

Note that, in Example 2.1, $\theta$ satisfies the condition $C$.

For every $x, y \in S$ and every $t_{1}, t_{2} \in[0,1]$ from condition $C$ we have

$$
\eta\left(y+t_{2} \eta(x, y), y+t_{1} \eta(x, y)\right)=\left(t_{2}-t_{1}\right) \eta(x, y),
$$

see [12] for details.

Proposition 3.1 Let $S \subseteq \mathbb{R}^{n}$ be an invex set with respect to $\eta: S \times S \rightarrow \mathbb{R}^{n}$ and $f: S \rightarrow \mathbb{R}$ is a function. Suppose that $\eta$ satisfies condition $C$ on $S$. Then, for every $x, y \in S$ the function $f$ is preinvex with respect to $\eta$ on $\eta$-path $P_{x v}$ if and only if the function $\varphi:[0,1] \rightarrow \mathbb{R}$ defined by

$$
\varphi(t):=f(x+\operatorname{t\eta }(y, x))
$$

is convex on $[0,1]$.

Proof Suppose that $\varphi$ is convex on $[0,1]$ and $z_{1}:=x+t_{1} \eta(y, x) \in P_{x v}, z_{2}:=x+t_{2} \eta(y, x) \in P_{x v}$. Fix $\lambda \in[0,1]$. Since $\eta$ satisfies condition $C$, by (13) we have

$$
\begin{aligned}
f\left(z_{1}+\lambda \eta\left(z_{2}, z_{1}\right)\right) & =f\left(x+\left((1-\lambda) t_{1}+\lambda t_{2}\right) \eta(y, x)\right) \\
& =\varphi\left((1-\lambda) t_{1}+\lambda t_{2}\right) \\
& \leq(1-\lambda) \varphi\left(t_{1}\right)+\lambda \varphi\left(t_{2}\right) \\
& =(1-\lambda) f\left(z_{1}\right)+\lambda f\left(z_{2}\right) .
\end{aligned}
$$

Hence, $f$ is preinvex with respect to $\eta$ on $\eta$-path $P_{x v}$. 
Conversely, let $x, y \in S$ and the function $f$ be preinvex with respect to $\eta$ on $\eta$-path $P_{x v}$. Suppose that $t_{1}, t_{2} \in[0,1]$. Then, for every $\lambda \in[0,1]$ we have

$$
\begin{aligned}
\varphi\left((1-\lambda) t_{1}+\lambda t_{2}\right) & =f\left(x+\left((1-\lambda) t_{1}+\lambda t_{2}\right) \eta(y, x)\right) \\
& =f\left(x+t_{1} \eta(y, x)\right)+\lambda \eta\left(x+t_{2} \eta(y, x), x+t_{1} \eta(y, x)\right) \\
& \leq \lambda f\left(x+t_{2} \eta(y, x)\right)+(1-\lambda) f\left(x+t_{1} \eta(y, x)\right) \\
& =\lambda \varphi\left(t_{2}\right)+(1-\lambda) \varphi\left(t_{1}\right) .
\end{aligned}
$$

Therefore, $\varphi$ is quasi-convex on $[0,1]$.

The following theorem is a generalization of Proposition 1 in [6].

Theorem 3.1 Let $S \subseteq \mathbb{R}^{n}$ be an open invex set with respect to $\eta: S \times S \rightarrow \mathbb{R}^{n}$. Assume that $\eta$ satisfies condition $C$. Suppose that for every $x, y \in S$ the function $f: S \rightarrow \mathbb{R}^{+}$is preinvex with respect to $\eta$ on $\eta$-path $P_{x v}$. Then, for every $a, b \in(0,1)$ with $a<b$ the following inequality holds,

$$
\begin{aligned}
& \left|\frac{1}{2} \int_{0}^{a} f(x+s \eta(y, x)) d s+\frac{1}{2} \int_{0}^{b} f(x+s \eta(y, x)) d s-\frac{1}{b-a} \int_{a}^{b}\left(\int_{0}^{s} f(x+t \eta(y, x)) d t\right) d s\right| \\
& \quad \leq \frac{b-a}{8}\{f(x+a \eta(y, x))+f(x+b \eta(y, x))\} .
\end{aligned}
$$

Proof Let $x, y \in S$ and $a, b \in(0,1)$ with $a<b$. Since $f$ is preinvex with respect to $\eta$ on $\eta$-path $P_{x v}$ by Proposition 3.1 the function $\varphi:[0,1] \rightarrow \mathbb{R}^{+}$defined by

$$
\varphi(t):=f(x+\operatorname{t\eta }(y, x))
$$

is convex on $[0,1]$. Now, we define the function $\phi:[0,1] \rightarrow \mathbb{R}^{+}$as follows

$$
\phi(t):=\int_{0}^{t} \varphi(s) d s=\int_{0}^{t} f(x+s \eta(y, x)) d s .
$$

Obviously for every $t \in(0,1)$ we have

$$
\phi^{\prime}(t)=\varphi(t)=f(x+t \eta(y, x)) \geq 0
$$

hence, $\left|\phi^{\prime}(t)\right|=\phi^{\prime}(t)$. Applying Theorem 1.1 to the function $\phi$ implies that

$$
\left|\frac{\phi(a)+\phi(b)}{2}-\frac{1}{b-a} \int_{a}^{b} \phi(s) d s\right| \leq \frac{(b-a)\left(\phi^{\prime}(a)+\phi^{\prime}(b)\right)}{8},
$$

and we deduce that (16) holds.

\section{Second-order differentiable functions}

In this section, we introduce some generalizations of Hermite-Hadamard-type inequality for functions whose second derivatives absolute values are preinvex. We begin with the following lemma (see Lemma 1 in [14] and Lemma 4 in [15]). 
Lemma 4.1 Let $A \subseteq \mathbb{R}$ be an open invex subset with respect to $\theta: A \times A \rightarrow \mathbb{R}$ and $a, b \in A$ with $\theta(a, b) \neq 0$. Suppose that $f: A \rightarrow \mathbb{R}$ is a differentiable function. If $f^{\prime \prime}$ is integrable on the $\theta$-path $P_{b c}, c=b+\theta(a, b)$ then, the following equality holds

$$
\begin{gathered}
\frac{f(b)+f(b+\theta(a, b))}{2}-\frac{1}{\theta(a, b)} \int_{b}^{b+\theta(a, b)} f(x) d x \\
=\frac{\theta(a, b)^{2}}{2} \int_{0}^{1} t(1-t) f^{\prime \prime}(b+t \theta(a, b)) d t .
\end{gathered}
$$

Proof Suppose that $a, b \in A$. Since $A$ is an invex set with respect to $\theta$, for every $t \in[0,1]$ we have $b+t \theta(a, b) \in A$. Integrating by parts implies that

$$
\begin{aligned}
& \int_{0}^{1} t(1-t) f^{\prime \prime}(b+t \theta(a, b)) d t \\
& \quad=\left[\frac{t(1-t) f^{\prime}(b+t \theta(a, b))}{\theta(a, b)}\right]_{0}^{1}-\frac{1}{\theta(a, b)} \int_{0}^{1}(1-2 t) f^{\prime}(b+t \theta(a, b)) d t \\
& \quad=\frac{f(b)+f(b+\theta(a, b))}{\theta(a, b)^{2}}-\frac{2}{(\theta(a, b))^{3}} \int_{b}^{b+\theta(a, b)} f(x) d x,
\end{aligned}
$$

which completes the proof.

Theorem 4.1 Let $A \subseteq \mathbb{R}$ be an open invex subset with respect to $\theta: A \times A \rightarrow \mathbb{R}$ and $\theta(a, b) \neq$ 0 for all $a \neq b$. Suppose that $f: A \rightarrow \mathbb{R}$ is a twice differentiable function on $A$. If $\left|f^{\prime \prime}\right|$ is preinvex on $A$ and $f^{\prime \prime}$ is integrable on the $\theta$-path $P_{b c}, c=b+\theta(a, b)$ then, the following inequality holds

$$
\begin{aligned}
& \left|\frac{f(b)+f(b+\theta(a, b))}{2}-\frac{1}{\theta(a, b)} \int_{b}^{b+\theta(a, b)} f(x) d x\right| \\
& \quad \leq \frac{\theta(a, b)^{2}}{24}\left(\left|f^{\prime \prime}(a)\right|+\left|f^{\prime \prime}(b)\right|\right) .
\end{aligned}
$$

Proof Suppose that $a, b \in A$. Since $A$ is an invex set with respect to $\theta$, for every $t \in[0,1]$ we have $b+t \theta(a, b) \in A$. By preinvexity of $\left|f^{\prime \prime}\right|$ and Lemma 4.1 we get

$$
\begin{aligned}
& \left|\frac{f(b)+f(b+\theta(a, b))}{2}-\frac{1}{\theta(a, b)} \int_{b}^{b+\theta(a, b)} f(x) d x\right| \\
& \quad=\left|\frac{\theta(a, b)^{2}}{2} \int_{0}^{1} t(1-t) f^{\prime \prime}(b+t \theta(a, b)) d t\right| \\
& \quad \leq \frac{\theta(a, b)^{2}}{2}\left[\int_{0}^{1} t(1-t)\left(t\left|f^{\prime \prime}(a)\right|+(1-t)\left|f^{\prime \prime}(b)\right|\right) d t\right] \\
& \quad=\frac{\theta(a, b)^{2}}{24}\left[\left|f^{\prime \prime}(a)\right|+\left|f^{\prime \prime}(b)\right|\right],
\end{aligned}
$$

which completes the proof.

The corresponding version for powers of the absolute value of the second derivative is incorporated in the following theorem. 
Theorem 4.2 Let $A \subseteq \mathbb{R}$ be an open invex subset with respect to $\theta: A \times A \rightarrow \mathbb{R}$ and $\theta(a, b) \neq 0$ for all $a \neq b$. Suppose that $f: A \rightarrow \mathbb{R}$ is a twice differentiable function on $A$ and $\left|f^{\prime \prime}\right|^{\frac{p}{p-1}}$ is preinvex on $A$, for $p>1$. If $f^{\prime \prime}$ is integrable on the $\theta$-path $P_{b c}, c=b+\theta(a, b)$ then, the following inequality holds

$$
\begin{aligned}
& \left|\frac{f(b)+f(b+\theta(a, b))}{2}-\frac{1}{\theta(a, b)} \int_{b}^{b+\theta(a, b)} f(x) d x\right| \\
& \quad \leq \frac{\theta(a, b)^{2}}{16}(\sqrt{\pi})^{\frac{1}{p}}\left(\frac{\Gamma(1+p)}{\Gamma\left(\frac{3}{2}+p\right)}\right)^{\frac{1}{p}}\left(\left|f^{\prime \prime}(a)\right|^{q}+\left|f^{\prime \prime}(b)\right|^{q}\right)^{\frac{1}{q}} .
\end{aligned}
$$

Proof By preinvexity of $\left|f^{\prime \prime}\right|$, Lemma 4.1 and using the well-known Hölder integral inequality, we get

$$
\begin{aligned}
& \left|\frac{f(b)+f(b+\theta(a, b))}{2}-\frac{1}{\theta(a, b)} \int_{b}^{b+\theta(a, b)} f(x) d x\right| \\
& \quad \leq \frac{\theta(a, b)^{2}}{2} \int_{0}^{1} t(1-t)\left|f^{\prime \prime}(b+\theta(a, b))\right| d t \\
& \quad \leq \frac{\theta(a, b)^{2}}{2}\left(\int_{0}^{1}\left(t-t^{2}\right)^{p} d t\right)^{\frac{1}{p}}\left(\int_{0}^{1}\left|f^{\prime \prime}(b+\theta(a, b))\right|^{q} d t\right)^{\frac{1}{q}} \\
& \quad \leq \frac{\theta(a, b)^{2}}{2}\left(\frac{2^{-1-2 p} \sqrt{\pi} \Gamma(1+p)}{\Gamma\left(\frac{3}{2}+p\right)}\right)^{\frac{1}{p}}\left(\int_{0}^{1}\left(t\left|f^{\prime \prime}(a)\right|^{q}+(1-t)\left|f^{\prime \prime}(b)\right|^{q}\right) d t\right)^{\frac{1}{q}} \\
& \quad=\frac{\theta(a, b)^{2}}{16}(\sqrt{\pi})^{\frac{1}{p}}\left(\frac{\Gamma(1+p)}{\Gamma\left(\frac{3}{2}+p\right)}\right)^{\frac{1}{p}}\left(\left|f^{\prime \prime}(a)\right|^{q}+\left|f^{\prime \prime}(b)\right|^{q}\right)^{\frac{1}{q}}
\end{aligned}
$$

which completes the proof.

A more general inequality is given using Lemma 4.1, as follows:

Theorem 4.3 Let $A \subseteq \mathbb{R}$ be an open invex subset with respect to $\theta: A \times A \rightarrow \mathbb{R}$ and $\theta(a, b) \neq 0$ for all $a \neq b$. Suppose that $f: A \rightarrow \mathbb{R}$ is a twice differentiable function on $A$ and $\left|f^{\prime \prime}\right|^{q}$ is preinvex on $A$, for $q>1$. If $f^{\prime \prime}$ is integrable on the $\theta$-path $P_{b c}, c=b+\theta(a, b)$ then, the following inequality holds

$$
\begin{aligned}
& \left|\frac{f(b)+f(b+\theta(a, b))}{2}-\frac{1}{\theta(a, b)} \int_{b}^{b+\theta(a, b)} f(x) d x\right| \\
& \leq \frac{\theta(a, b)^{2}}{12}\left(\frac{1}{2}\right)^{\frac{1}{q}}\left(\left|f^{\prime \prime}(a)\right|^{q}+\left|f^{\prime \prime}(b)\right|^{q}\right)^{\frac{1}{q}} .
\end{aligned}
$$

Proof By preinvexity of $\left|f^{\prime \prime}\right|$, Lemma 4.1 and using the well-known weighted power mean inequality, we get

$$
\begin{gathered}
\left|\frac{f(b)+f(b+\theta(a, b))}{2}-\frac{1}{\theta(a, b)} \int_{b}^{b+\theta(a, b)} f(x) d x\right| \\
\leq \frac{\theta(a, b)^{2}}{2} \int_{0}^{1} t(1-t)\left|f^{\prime \prime}(b+\theta(a, b))\right| d t
\end{gathered}
$$




$$
\begin{aligned}
& \leq \frac{\theta(a, b)^{2}}{2}\left(\int_{0}^{1}\left(t-t^{2}\right) d t\right)^{1-1 / q}\left(\int_{0}^{1}\left(t-t^{2}\right)\left|f^{\prime \prime}(b+\theta(a, b))\right|^{q} d t\right)^{1 / q} \\
& \leq \frac{\theta(a, b)^{2}}{2}\left(\frac{1}{6}\right)^{1-1 / q}\left(\int_{0}^{1}\left(t-t^{2}\right)\left[t\left|f^{\prime \prime}(a)\right|^{q}+(1-t)\left|f^{\prime \prime}(b)\right|^{q}\right] d t\right)^{1 / q} \\
& \leq \frac{\theta(a, b)^{2}}{2}\left(\frac{1}{6}\right)^{1-1 / q}\left(\frac{1}{12}\left(\left|f^{\prime \prime}(a)\right|^{q}+\left|f^{\prime \prime}(b)\right|^{q}\right)\right)^{1 / q} \\
& =\frac{\theta(a, b)^{2}}{12}\left(\frac{1}{2}\right)^{\frac{1}{q}}\left(\left|f^{\prime \prime}(a)\right|^{q}+\left|f^{\prime \prime}(b)\right|^{q}\right)^{\frac{1}{q}}
\end{aligned}
$$

which completes the proof.

\section{Competing interests}

The authors declare that they have no competing interests.

\section{Authors' contributions}

All authors conceived of the study, and participated in the design and coordination. All authors read and approved the final manuscript.

\section{Author details}

${ }^{1}$ Department of Mathematics, Lorestan University, P.O. Box 465, Khoramabad, Iran. ${ }^{2}$ School of Engineering and Science, Victoria University, P.O. Box 14428, Melbourne City, VIC 8001, Australia. ${ }^{3}$ School of Computational and Applied Mathematics, University of the Witwatersrand, Private Bag 3, Johannesburg, Wits 2050, South Africa.

Received: 6 August 2011 Accepted: 28 May 2012 Published: 29 October 2012

\section{References}

1. Dragomir, SS: Two mappings on connection to Hadamard's inequality. J. Math. Anal. Appl. 167, 49-56 (1992)

2. Dragomir, SS: On Hadamard's inequalities for convex functions. Math. Balk. 6, 215-222 (1992)

3. Dragomir, SS, Pecaric, JE, Sandor, J: A note on the Jensen-Hadamard inequality. Anal. Numér. Théor. Approx. 19, 29-34 (1990)

4. Dragomir, SS, Pecaric, JE, Persson, LE: Some inequalities of Hadamard type. Soochow J. Math. 21, 335-341 (1995)

5. Dragomir, SS, Agarwal, RP: Two inequalities for differentiable mappings and applications to special means of real numbers and to trapezoidal formula. Appl. Math. Lett. 11, 91-95 (1998)

6. Ion, DA: Some estimates on the Hermite-Hadamard inequality through quasiconvex functions. Ann. Univ. Craiova, Math. Comput. Sci. Ser. 34, 82-87 (2007)

7. Hanson, MA: On sufficiency of the Kuhn-Tucker conditions. J. Math. Anal. Appl. 80, 545-550 (1981)

8. Weir, T, Mond, B: Preinvex functions in multiple objective optimization. J. Math. Anal. Appl. 136, 29-38 (1998)

9. Noor, MA: Hermite-Hadamard integral inequalities for log-preinvex functions. J. Math. Anal. Approx. Theory 2 , 126-131 (2007)

10. Noor, MA: On Hadamard integral inequalities involving two log-preinvex functions. J. Inequal. Pure Appl. Math. 8, 1-6 (2007)

11. Antczak, T: Mean value in invexity analysis. Nonlinear Anal. 60, 1471-1484 (2005)

12. Yang, XM, Li, D: On properties of preinvex functions. J. Math. Anal. Appl. 256, 229-241 (2001)

13. Mohan, SR, Neogy, SK: On invex sets and preinvex function. J. Math. Anal. Appl. 189, 901-908 (1995)

14. Alomari, M, Drus, M, Dragomir, SS: New inequalities of Hermite-Hadamard type for functions whose second derivatives absolute values are quasi-convex. Tamkang J. Math. 41, 353-359 (2010)

15. Dragomir, SS, Pearce, CEM: Selected Topics on Hermite-Hadamard Inequalities and Applications. RGMIA Monographs. Victoria University, Melbourne (2000). http://ajmaa.org/RGMIA/monographs.php 